This document is published in:

Experimental Mechanics, (2014), 54 (6), 1099-1109.

DOI: http://dx.doi.org/10.1007/s11340-014-9860-8

(C)2014 Society for Experimental Mechanics 


\title{
Dynamic Necking of Notched Tensile Bars: An Experimental Study
}

\author{
D. Rittel ${ }^{1.2}$, Y. Rotbaum ${ }^{1}$, J.A. Rodríguez-Martínez ${ }^{1.2 . *}$, D. Sory ${ }^{1.3}$, R. Zaera ${ }^{2}$ \\ 1. Faculty of Mechanical Engineering, Technion, 32000 Haifa, Israel \\ 2. Department of Continuum Mechanics and Structural Analysis, University Carlos III of Madrid, \\ Avda. de la Universidad, 30, 28911 Leganés, Madrid, Spain \\ *e-mail: jarmarti@ing.uc3m.es \\ 3. Ecole Centrale des Arts et Metiers, Bruxelles, Belgium
}

\begin{abstract}
The mechanics of necking inception in dynamically-stretched notched specimens have been investigated. For that task, a systematic experimental campaign of quasi-static and dynamic tensile tests on martensitic steel specimens has been conducted. Samples with and without notches have been considered. Unlike the quasi-static tests, the dynamically-tested notched samples revealed that, under certain loading conditions, flow localization may develop away from the groove. The experimental results presented in this investigation show that the presence of sharp geometrical imperfections in ductile materials subjected to dynamic loading does not necessarily dictate the necking and fracture locus.
\end{abstract}

Keywords Dynamic necking · Notch · Tensile tests · Weakest link

\section{Introduction}

Structural elements often exhibit abrupt changes in the crosssectional area or disruptions of the smooth surface. Grooves, fillets, holes, sharp corners or threads are all examples of geometric discontinuities causing the solid to experience a local increase in the intensity of the stress field which reaches much larger magnitudes than does the average stress over the section, as shown by theoretical analyses and experimental measurements. Notch or stress concentrator (in regard to their geometric features or to their internal force distribution, re-spectively) is a general term meaning any or all of the above. A theoretical analysis of the role of stress concentrators was firstly highlighted by Inglis [1] who gave a stress concentration factor for an elliptical defect.
Later on, Neuber [2, 3] a n d Peterson [4, 5] were concerned with predicting failure in metallic components, and developed the classical explanations for notch effects. Many analytical equations expressing the distribution of elastic-plastic stress and strain concentration fields at a notch were developed by these two authors. Because of the importance in structural design of notched tension members, the stress-concentration effects on them are of prac-tical interest. The problem has therefore received considerable attention since the pioneering works of Preuss [6], Cocker, [7], Cocker et al. [8] and Howland [9], up to the more recent due to Strandberg [10] and Zappalorto and Lazzarin [11]. Notch effects are continuously re-examined by employing experi-mental, analytical and numerical methods.

A considerable amount of work has been achieved to determine the quasi-static stress concentration factors for common geometrical discontinuities. However, although designers are concerned about high-speed loading applications, relatively few studies have been carried out to examine the behavior of notched bars under dynamic tensile loading. The understanding and documentation of dynamic stress concentration factors has been considered an important field for many areas of mechanical engineering, including crashworthiness, high-speed impact, and transportation of hazardous materials [12]. Experimental [13, 14] and numerical $[12,15]$ techniques have been used to analyze the influence of notch geometry and loading conditions on the differences between quasi-static and dynamic elastic stress concentration factors.

What definitely makes the analysis of notches relevant is that the majority of failures, in both quasi-static and impact 
conditions emanate from stress concentrators. Therefore, the underlying idea behind all these studies is the consideration of the notch as a structural weak point, hence naturally regarded as a definite source of failure. However, and bearing in mind the undisputed relevance of all the aforementioned analyses for the design of structural elements, it is worth reassessing the problem for dynamic loading conditions. Stated otherwise: Would it be possible to find a loading condition under which the fracture locus will skip the notch? Based on the experience gained in quasi-static conditions throughout over a century, it seems difficult to respond positively to this question. However, under suitable impact loading, we found a manifest exception to this rule, specifically when wave propagation is strongly present in the loading process. Impact tests designed to characterize the dynamic behavior of materials tend to avoid any phenomenon, usually related to wave propagation, that may hide the real stress-strain characteristic of the material. Some distinctive examples are peaks in the input waves, that may be damped by pulse shapers [16], or lack of equilibrium, that may be prevented by using short specimens. Alternatively, the propagation of stress waves in the axial direction of the specimen is precluded considering appropriate symmetries in the geometric and loading features of the tests, like in the radial expansion of a ring [17, 18$]$.

In this work, a study has been conducted to assess the effects a stress concentrator may have on a dynamically loaded specimen, in presence of stress waves. For that task, a systematic experimental campaign of quasi-static and dynamic tensile tests of martensitic steel specimens has been conducted. Samples without and with notches, at different locations along the specimen's gauge, were considered. The dynamic experiments revealed that a neck (and subsequent failure surface) may develop away from the notch. The presence of a weak point in the structure will not necessarily dictate the failure locus at high loading rates. Moreover, for identical applied velocities and notch position, the results are quite repeatable and predictable thus strengthening the idea that necking location is deterministic and dictated by dynamic effects, namely stress waves and inertia.

\section{Experimental Setup}

The material of this study is a hardened 15-5 PH steel (condition A), supplied as a $12.7 \mathrm{~mm}$ diameter bar, and tested in the as-received condition. This material is a high-strength fully martensitic steel with additional strengthening due to precipitation. Tensile cylindrical specimens with end threads were machined from the bar. The specimens' dimensions are shown in Fig. 1 and Table 1. Two types of specimens were manufactured: smooth (no notch) and notched. The geometry and dimensions of the notches were carefully checked for each specimen after machining using a profilometer, which revealed a natural scatter from one specimen to the other. Only those notches which fulfilled the geometrical requirements listed in Table 2 were considered as valid specimens. The notches were machined alternatively at a distance $\mathrm{M}, \mathrm{N}$ or $\mathrm{L}$ from the loaded end of the sample, as shown in Fig. 1.

Quasi-static and dynamic tests were conducted using both smooth and notched samples, as follows:

- Quasi-static tests: The quasi-static tensile specimens were tested using a servo-hydraulic testing machine (MTS 810) under displacement control. A laser optical extensometer (LE-05, EIR) was used for the axial strain measurements. A total of 3 smooth and 3 notched specimens were tested.

- Dynamic tests: The dynamic tensile specimens were tested in a $12.7 \mathrm{~mm}$ diameter tensile Kolsky apparatus (Split Hopkinson Tensile Bar), made of hardened 17-4 PHsteel, in which the end of the incident bar is loaded by a $320 \mathrm{~mm}$ long gas-launched hollow cylindrical impactor. A rotating mirror high speed camera (Cordin 530) was synchronized with the incident bar signals to capture the evolution of the specimen and neck's development during the dynamic tensile tests. The tested impact velocities ranged from 10 to $25 \mathrm{~m} / \mathrm{s}$. The applied velocities are determined from the measured incident, reflected and transmitted pulses, as for any other standard test with the Split Hopkinson Tensile Bar. It should be noted that the gauge length is much longer than the diameter of the

Fig. 1 Geometry of the tensile specimens

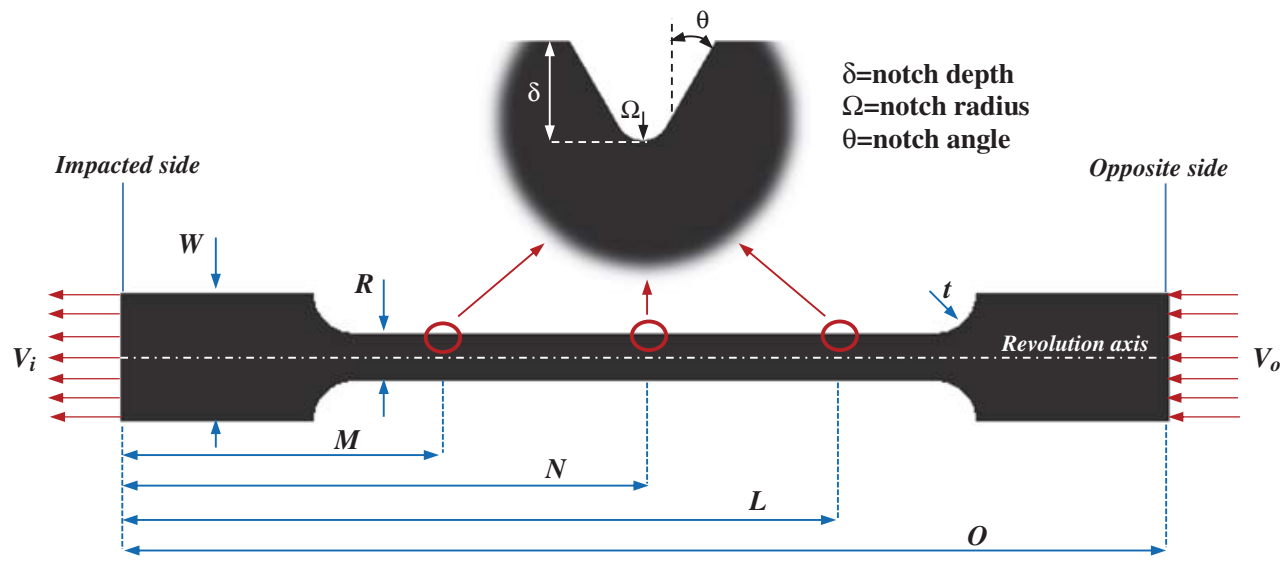


Table 1 Dimensions of the sample (mm)

\begin{tabular}{lllllll}
\hline $\mathrm{M}$ & $\mathrm{N}$ & $\mathrm{L}$ & $\mathrm{O}$ & $\mathrm{R}$ & $\mathrm{t}$ & $\mathrm{W}$ \\
\hline 26.5 & 32.5 & 38.5 & 65.0 & 3.0 & 2.5 & 8.0
\end{tabular}

specimens, which allows for nearly one-dimensional wave propagation within the sample. Let us remark that stress wave's propagation and interaction play a key role on necking inception as further discussed in forthcoming sections of the paper. A total of 5 smooth and 13 notched specimens were tested.

\section{Quasi-Static Tests}

Typical true stress-strain curves for smooth and notched samples under quasi-static loading are shown in Fig. 2. The 15-5 $\mathrm{PH}$ steel possesses a rather high yield strength, $\sigma_{y} \sim 1,100 \mathrm{MPa}$, and very limited strain hardening. These are common characteristics shared by many commercial martensitic steels.

It is well known that under quasi-static loading, and therefore an equilibrated specimen, the weakest section dictates the fracture location. Such behavior was indeed observed in the experiments:

- Smooth samples: Fracture was preferentially located close to the middle of the gauge, although it exhibited certain variability. Under ideal conditions the necking location corresponds to the middle of the gauge due to symmetric loading and boundary conditions. However, due to the presence of natural (material) or induced (geometrical) flaws, the weakest section may be occasionally shifted leading to fracture closer to the sample ends, as illustrated in Fig. 2.

- Notched samples: fracture was located in the notch for all the tested specimens. The notch was the seed for necking nucleation, accelerating flow localization. The groove reduces the cross-sectional area of the sample and concentrates stresses, therefore acting as the weakest point irrespective of its location in the gauge. Necking inception occurred as soon as the material underwent plasticity. The macroscopic necking strain, here defined as $\varepsilon_{n}=\left.\varepsilon\right|_{\frac{d \sigma}{d \varepsilon}=0}$, of the notched samples was much smaller, $\varepsilon_{n} \sim 0.01$, than in the case of the smooth samples, $\varepsilon_{n} \sim 0.04$, as shown in Fig. 2.

Table 2 Dimensions of the notch

\begin{tabular}{lll}
\hline$\delta(\mathrm{mm})$ & $\Omega(\mathrm{mm})$ & $\theta$ \\
\hline $0.15 \pm 0.002$ & $0.05 \pm 0.005$ & $55^{\circ} \pm 5^{\circ}$ \\
\hline
\end{tabular}

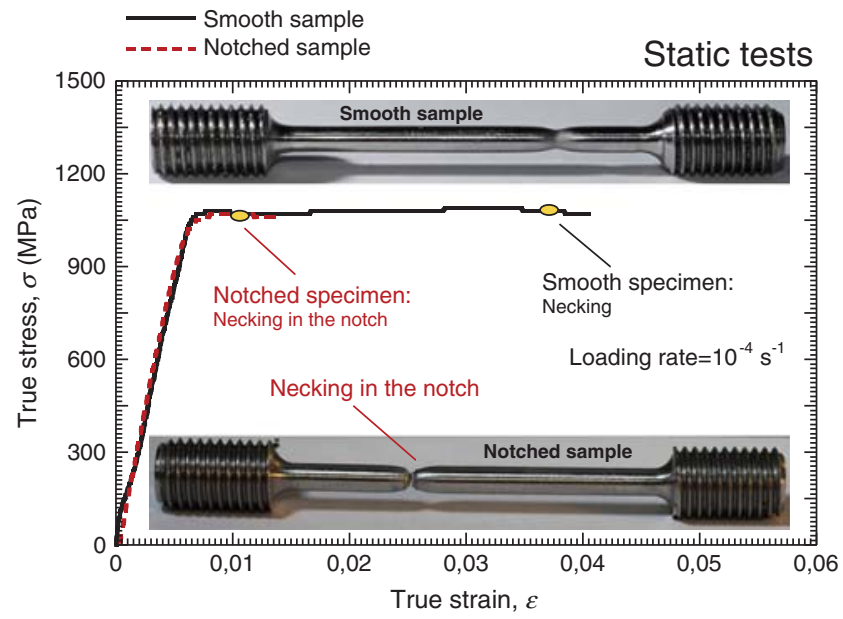

Fig. 2 Typical true stress-strain curves for smooth and notched samples under quasi-static loading. Note the early necking of the notched specimen

These results, which represent an (another) experimental verification of the weakest link theory in quasi-statics, trigger the following questions:

- Are the quasi-static observations reported here applicable to the dynamic loading case? Can one automatically generalize the weakest point approach to cases where stress wave loading and inertia play a dominant role?

\section{Dynamic Tests}

\section{Smooth Specimens}

Table 3 lists the results of the dynamic tensile tests carried out on smooth specimens. As mentioned in Osovski et al. [19], one should note that the specimen is not rigidly clamped, which results in a small, yet non-negligible, velocity on the transmitted side. Before analyzing the experimental results, it should be noted that loading velocity is directly related to the stress wave induced in the sample (one may think here about

Table 3 Summary of the dynamic tensile tests carried out on smooth specimens

\begin{tabular}{llll}
\hline Specimen & $\begin{array}{l}\text { Input velocity, } \\
\mathrm{V}_{\mathrm{i}}(\mathrm{m} / \mathrm{s})\end{array}$ & $\begin{array}{l}\text { Output velocity, } \\
\mathrm{V}_{\mathrm{o}}(\mathrm{m} / \mathrm{s})\end{array}$ & Neck location \\
$\# 1$ & 15 (average) & 0.85 (average) & Impacted side \\
$\# 2$ & 13 (average) & 0.85 (average) & Impacted side \\
$\# 3$ & 12 (average) & 0.85 (average) & Impacted side \\
$\# 4$ & 18 (average) & 1.00 (average) & Opposite side \\
$\# 5$ & 17 (average) & 1.00 (average) & Opposite side \\
\hline
\end{tabular}


the one dimensional wave propagation theory). Therefore, behind the term "loading velocity" repeatedly invoked in the paper resides the magnitude of the stress wave induced in the specimen by the impact.

Table 3 and Fig. 3 show that when the incident velocity remains equal or smaller than $15 \mathrm{~m} / \mathrm{s}$, the neck forms on the impacted side, whereas at higher velocities, it now forms on the opposite side. This trend has been found and thoroughly discussed by the authors in previous works [19, 20]. The location of the neck is dictated by the loading velocity, which in turn controls the processes of propagation and interaction of stress waves. Interaction of waves triggers a heterogeneous distribution of plastic strains along the gauge of the sample, leading to necking inception away from the center of the specimen. Note that, in the absence of results for $16 \mathrm{~m} / \mathrm{s}$, one can speculate that this velocity is the pivot at which the neck location switches from impacted to opposite side. Altogether, one finds that it is quite difficult to exactly pinpoint the value of this transition velocity, as the transition itself is quite

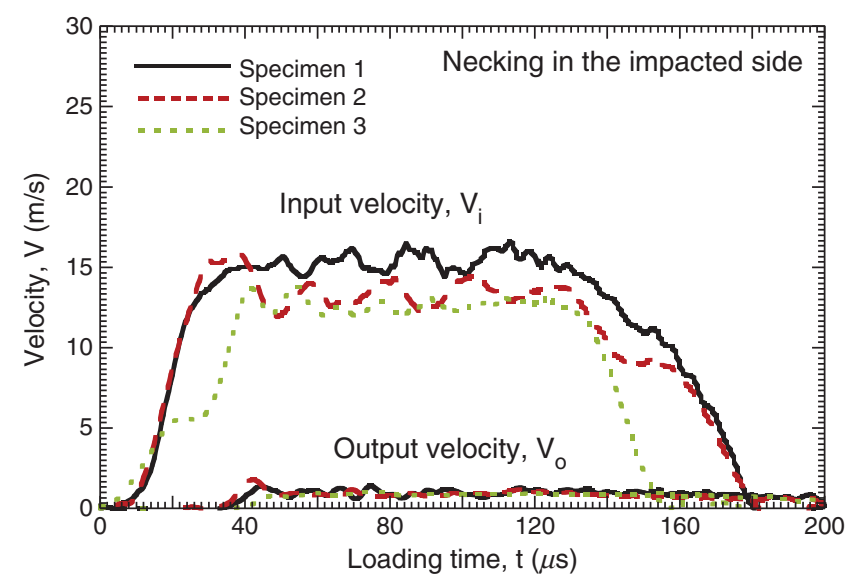

(a)

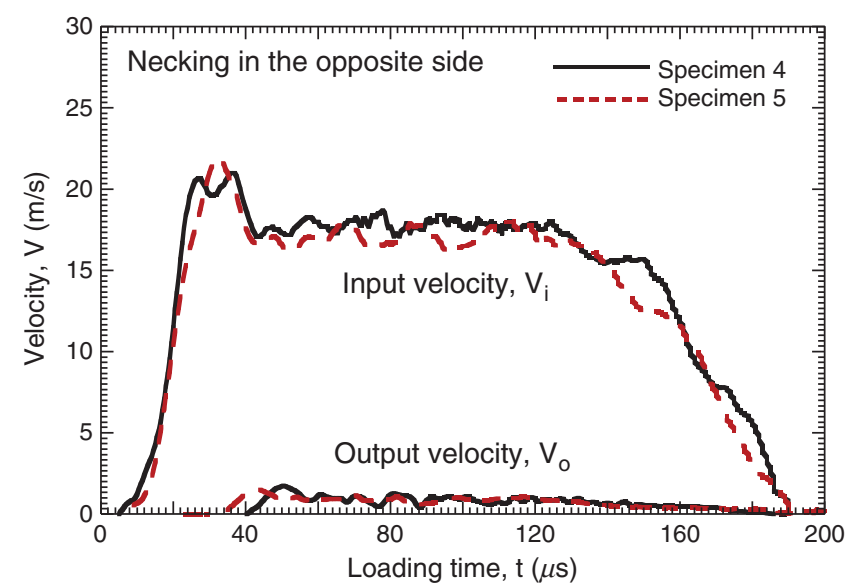

(b)

Fig. 3 Input and output velocity profiles of the dynamic tensile tests carried out on smooth specimens. a Tests for which the neck is located in the impacted side, $\mathbf{b}$ tests for which the neck is located in the opposite side abrupt as a sign of high sensitivity of the neck location to the applied loading (magnitude of the stress wave induced by the impact) and boundary conditions.

Typical velocity profiles are shown in Fig. 3, for which one can note that they all look relatively similar, without any noticeable feature, other than the magnitude of the input velocity (magnitude of the stress wave induced by the impact), that could cause the observed jump in neck location. One should also note that those results all indicate the high level of reproducibility of the observations as to neck location. These results also reinforce previous conclusions found in Osovski et al. [19], Sørensen and Freund [21] and Rodríguez-Martínez et al. [22] as to the deterministic character of the neck location in dynamic tensile and ring expansion tests, respectively.

The corresponding broken specimens are shown in Fig. 4. One noticeable observation is that, irrespective of the location of the neck (impacted or opposite side), the absolute distance of the neck from the fillet is remarkably similar for all the tested specimens.

\section{Notched Specimens}

Table 4 lists all the representative dynamic tests that were carried out on centrally notched specimens (notch location $\mathrm{N}$ in Fig. 1). The velocity profiles are shown in Fig. 5 for each reported experiment. As for the case of the smooth samples, they all look very similar in shape, except for the higher velocity tests for which an inertial peak is observed, prior to reaching a stable input velocity. Such peak velocities are listed in Table 4. The development of an inertial peak is mostly related to the nature of the test (in which severe accelerations are applied to the system) and the high length to diameter ratio of the samples. As previously mentioned for the dynamic experiments on the smooth

Table 4 Summary of the dynamic tensile tests carried out on centrally notched specimens

\begin{tabular}{llll}
\hline Specimen & $\begin{array}{l}\text { Input velocity, } \\
\mathrm{V}_{\mathrm{i}}(\mathrm{m} / \mathrm{s})\end{array}$ & $\begin{array}{l}\text { Output velocity, } \\
\mathrm{V}_{\mathrm{o}}(\mathrm{m} / \mathrm{s})\end{array}$ & Neck location \\
\hline$\# 6$ & 15.0 (average) & 1.1 (average) & Impacted side \\
$\# 7$ & 15.0 (average) & 1.1 (average) & Impacted side \\
$\# 8$ & 25.0 (peak) & 1.3 (average) & Opposite side \\
$\# 9$ & 21.7 (peak) & 0.8 (average) & Opposite side \\
$\# 10$ & 25.0 (peak) & 0.8 (average) & Opposite side \\
$\# 11$ & 18.8 (peak) & 0.9 (average) & Notch \\
$\# 12$ & 13.3 (average) & 0.9 (average) & Notch \\
$\# 13$ & 16.9 (average) & 0.9 (average) & Notch \\
$\# 14$ & 23.6 (peak) & 0.9 (average) & Notch \\
\hline
\end{tabular}


samples, the dynamic tests on notched specimens reveal a highly unstable position of the neck that most likely results from a high sensitivity to the loading (magnitude of the stress wave induced by the impact) and boundary conditions applied in the tests. But most of all, the present results clearly show, for the first time to the best of the authors' knowledge, that the presence of a notch (weak or weakest point) does not necessarily dictate the location of the neck, the latter being capable of "ignoring" the notch, Table 4 and Fig. 6. The processes of propagation and interaction of stress waves boost plastic strains in the smooth section to a critical level which overrules any stress concentration effects. This observation stands at odds with neck location in quasi-static testing.

While the output velocities are all of the same order of magnitude, the results clearly indicate that the neck location jumps from the impacted to the opposite side as the input velocity increases, Fig. 5. Basically, specimens 6-10 develop a neck at the same location than do smooth specimens for similar impact velocities.

The results also show a "twilight velocity zone" in which the neck is located in the notch (specimens 11-14), and this zone covers both the lower and higher velocities applied in the tests. These specimens do not reveal a specific pattern. However, one interesting observation is shown in Fig. 7. For this specimen (\# 14), fracture indeed occurred in the notch but a second diffuse neck was clearly observed on the opposite side, as would be expected for a smooth specimen tested at a similar velocity (within the higher velocities tested). Such observations are quite rare, from an experimental point of view, although they can be predicted by numerical simulations [20, 23]. One can therefore postulate that the "expected" location of the diffuse neck in specimen 14, together with the apparent lack of consistent pattern for specimens 11-14, all suggest some variability in the notch depth as reported in Table 2.
While the sensitivity of necking to notch geometry has not been investigated here, it appears to be quite high and this issue certainly deserves additional investigation.

To gain further experimental insight into the notch-neck location interplay, we consider specimen 5 that was impacted at about $17 \mathrm{~m} / \mathrm{s}$ and failed on the opposite side. Additional tests were carried out on same geometry specimens, similar applied velocity $(\sim 16 \mathrm{~m} / \mathrm{s})$, but this time, the notch was machined at $1 / 3$ of the gauge length (locations $M$ and $L$ in Fig. 1). A total of 4 experiments were carried out, namely 2 specimens with the notch at $1 / 3$ of the gauge length on the impacted side (location $\mathrm{M}$ ) and the other 2 with the notch at $2 / 3$ of the gauge length, that is at $1 / 3$ of the gauge length on the transmitted side (location L), Table 5. The applied velocity profiles are shown in Fig. 8, showing that apart from the notch location, the boundary conditions were highly repeatable. As expected for this value of the input velocity (magnitude of the stress wave induced by the impact), necking and fracture take place close to the opposite side of the specimen. Consequently, when the notch was on that side, fracture occurred in the notch, but when it was placed on the incident side, necking still occurred close to the opposite side, therefore outside the notch. Those tests are quite instructive since they first of all strengthen the point that, for identical applied velocities, the results are quite repeatable and predictable. Moreover the results show once again that the presence of the notch in the specimen may or may not affect the location of the neck and subsequent fracture, indicating that the presence of a weakest point in the structure will not necessarily dictate the failure locus at high loading rates, Fig. 9. It is assumed that this behavior is caused by the major role that inertia and stress waves play in the response of the material under impact loading. As previously mentioned, due to wave interaction processes, the plastic strains may apparently build up to critical levels in the smooth section which overrule any stress concentration effects.

Fig. 4 Tested smooth specimens

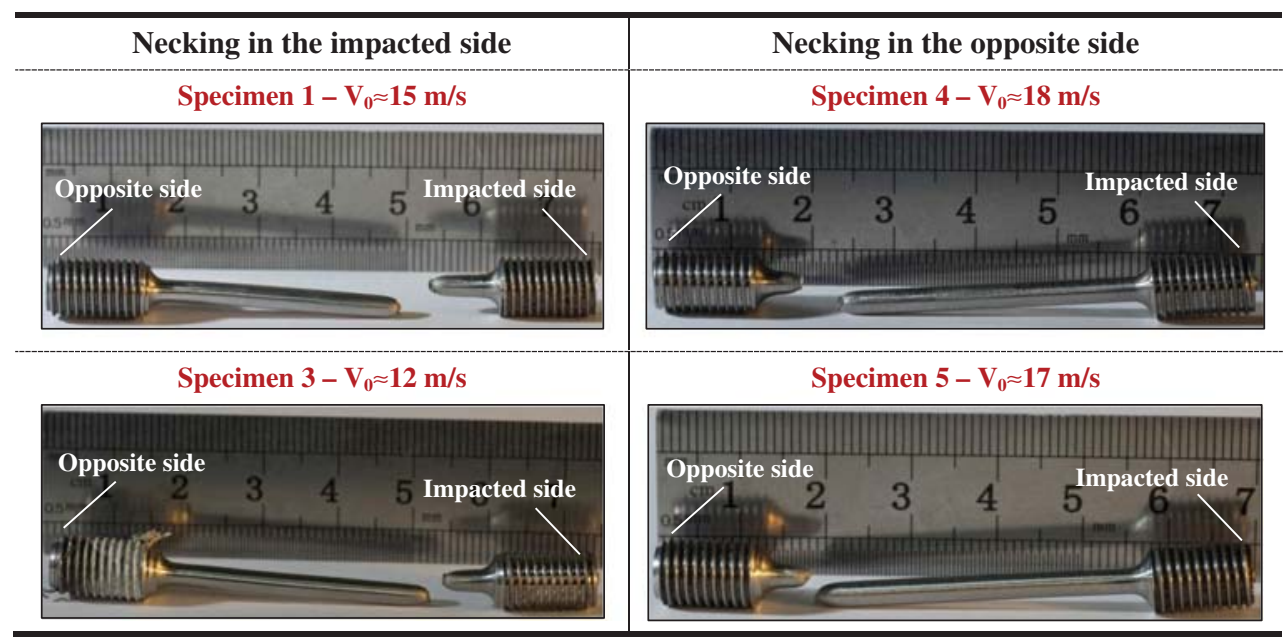




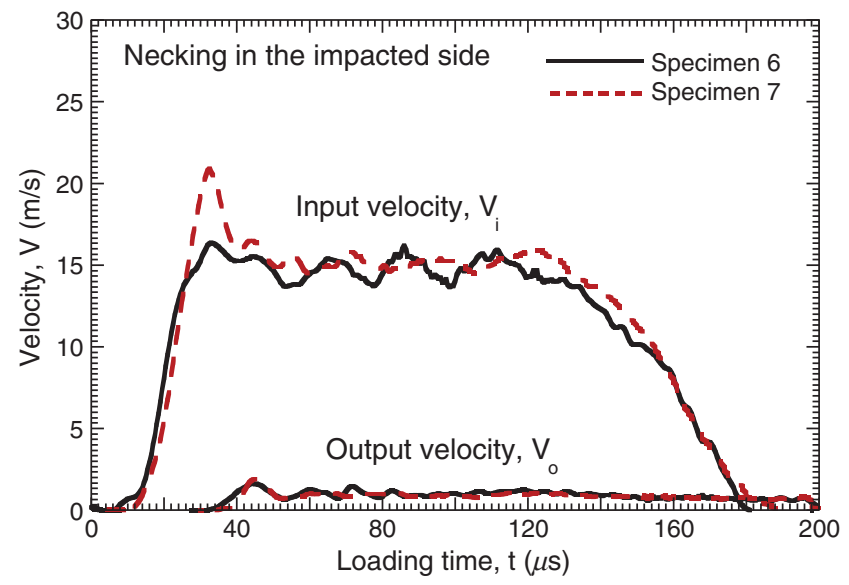

(a)

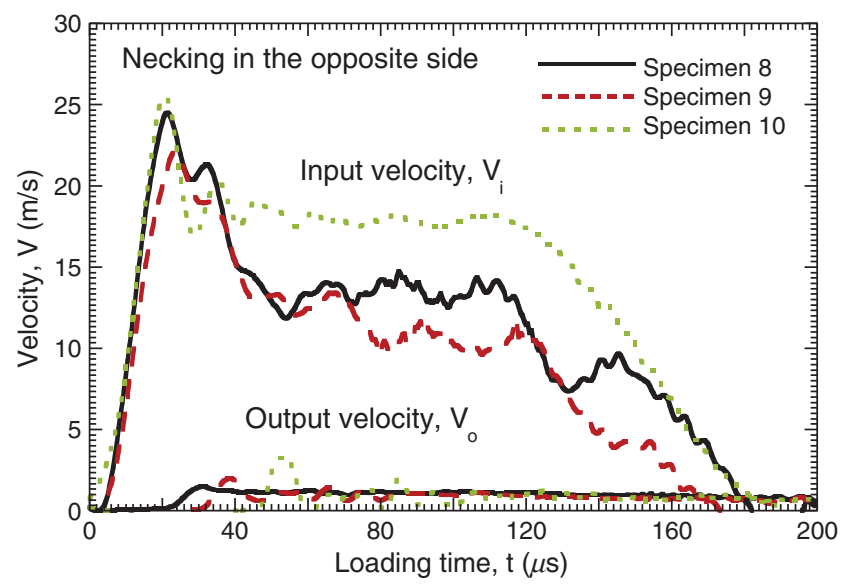

(b)

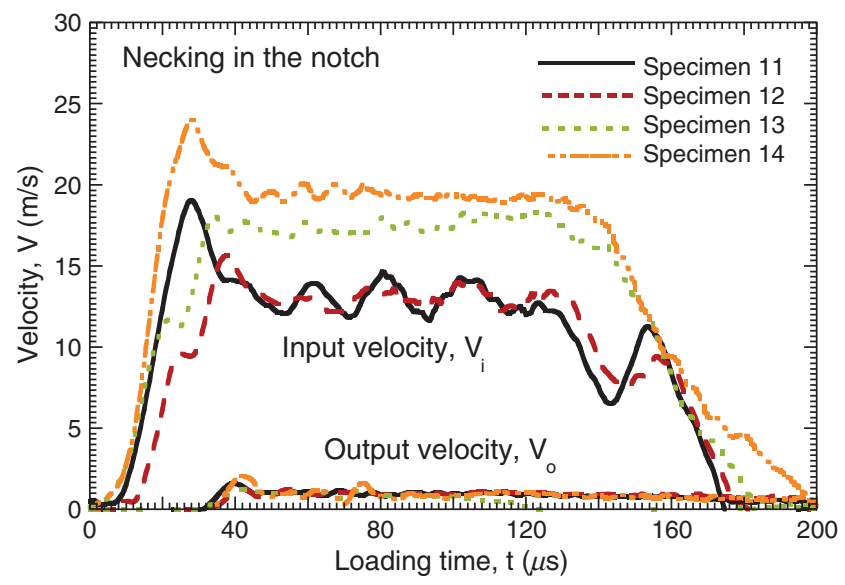

(c)

Fig. 5 Input and output velocity profiles of the dynamic tensile tests carried out on centrally notched specimens. a Tests for which the neck is located in the impacted side, $\mathbf{b}$ tests for which the neck is located in the opposite side, $\mathbf{c}$ tests for which the neck is located in the notch

One last point concerns the overall strain to fracture of the notched vs. smooth tested specimens, as it is important to assess whether the presence of a notch in the structure affects its energy absorption capacity. The appendix section presents the high speed recordings of the specimens' deformation until full neck formation (and fracture in some cases). Given the oscillations of the velocity measurements intrinsic to the dynamic character of the tests, one can calculate the macroscopic elongation of the specimen until inception of the neck. The recordings shown in the appendix show that the variability of the calculated macroscopic ductilities (sample deformation at the onset of the necking) is relatively small, which suggests that the presence of the notch in the specimen does not affect, to a first extent, the macroscopic ductility of the tested specimens. For all the tested specimens, with and without notches, the macroscopic strain to necking varies between $4 \%$ and $5 \%$ (see Appendix section).

\section{Discussion}

This paper is of an experimental nature, aiming to assess the effects that a stress concentrator (notch) may have on a dynamically tensile tested structure. Before discussing further the main outcomes of this work, one should emphasize the context of the study with respect to the weakest link theory that was repeatedly invoked here. The weakest link theory was originally developed for brittle fracture in the quasi-static regime whereas the present work concerns dynamic failure of a ductile material. Hence, one of the goals of this work was to assess the extent to which the weakest link approach can be generalized to dynamic ductile failure. In the present context, one should also mention recent work about dynamic tensile loading of smooth specimens made of ductile steel [19]. In that study, emphasis was put on the deterministic (thus predictable) nature of the neck location, showing the latter could be reproduced by a numerical model provided care was taken to apply the prevailing boundary conditions. In a sense, the present work is a continuation of our previous work, with the emphasis placed this time on the influence of a geometrical discontinuity (notch) in the specimen.

First of all, one should note that the notches of this study cannot be considered as shallow defects. On the contrary, they are relatively short-wavelength, sharp and deep imperfections, whose depth reaches 0.1 of the specimen diameter.

Experiments of a similar nature have not been previously reported in the literature to the best authors' knowledge, perhaps because one natural assumption would be that failure always occurs in the stress concentrator, based on the quasistatic experience. However, the present results show that it is not necessarily so. Depending on the applied input velocity (magnitude of the stress wave induced by the impact), while the output velocities remain at a comparable level in all tests, one observes that the neck can locate on the impacted side at 
Fig. 6 Tested centrally notched specimens

Fig. 7 Post mortem observation. Specimen 14. Fracture in the notch and secondary (diffuse) neck in the opposite side
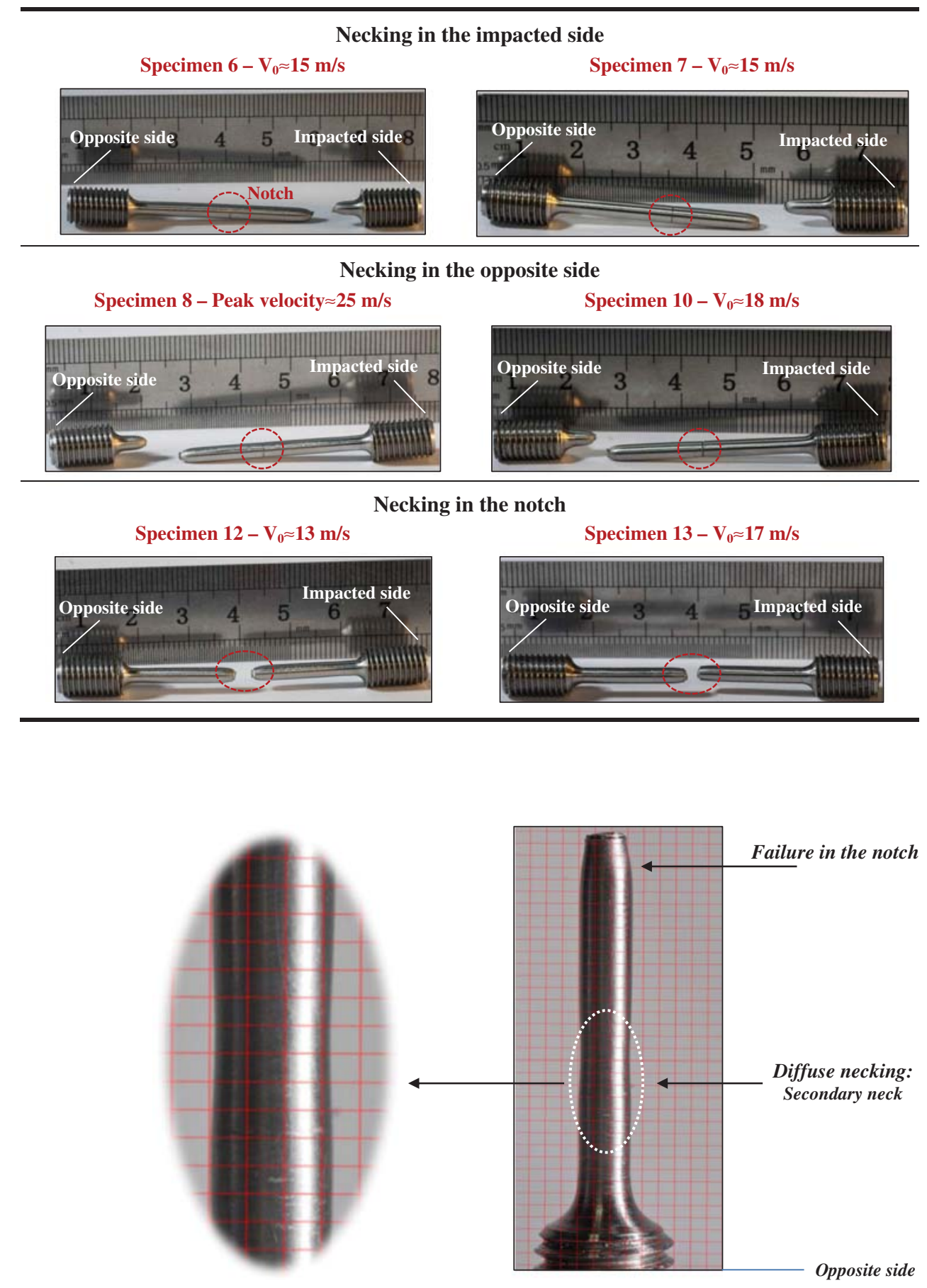

Table 5 Summary of the dynamic tensile tests carried out on sided notched specimens

\begin{tabular}{lllll}
\hline Specimen & Input velocity, $\mathrm{V}_{\mathrm{i}}(\mathrm{m} / \mathrm{s})$ & Output velocity, $\mathrm{V}_{\mathrm{o}}(\mathrm{m} / \mathrm{s})$ & Notch location & Neck location \\
\hline$\# 15$ & 15.0 (average) & 1.0 (average) & $\mathrm{M}$ & Opposite side \\
$\# 16$ & 15.0 (average) & 1.0 (average) & $\mathrm{L}$ & Opposite side-Notch \\
$\# 17$ & 15.0 (average) & 1.0 (average) & $\mathrm{M}$ & Opposite side \\
$\# 18$ & 15.0 (average) & 1.0 (average) & $\mathrm{L}$ & Opposite side-Notch \\
\hline
\end{tabular}




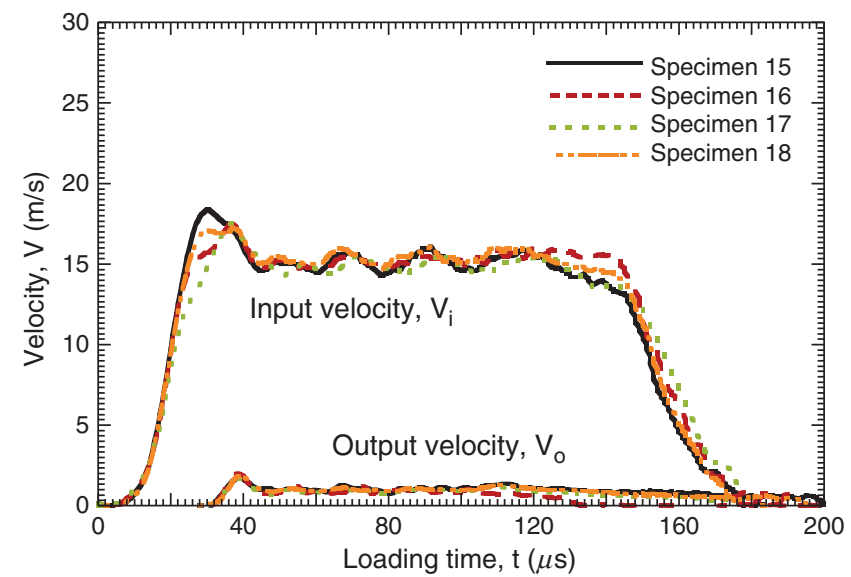

Fig. 8 Input and output velocity profiles of the dynamic tensile tests carried out on sided notched specimens

the lower tested velocity, ending on the opposite side of the specimen for the higher tested velocities. Yet, some specimens fractured in the notch without apparent pattern in terms of velocity, a fact that seems to be related to some variability in the groove geometry. One should note that the reported trend in neck location is only partial, as the range of applied velocities did not exceed some $25 \mathrm{~m} / \mathrm{s}$ while a full picture would be obtained had higher velocities been applied. Unfortunately, the current experimental setup did not allow for reaching much higher velocities. However, the reported results nevertheless indicate a clear trend in the variation of neck location with respect to the prescribed impact velocity (magnitude of the stress wave induced by the impact), and most of all that necking does not necessarily take place in the notch. In other words, the number of observed exceptions to the "anticipated rule" of notch induced necking is largely sufficient to question the latter assumption. It appears that the processes of wave propagation and interaction cause high gradients of stress along the sample. As a consequence, plastic strains pile up to a critical level in the smooth section which overrides any stress concentration effects.

The results also show that, to a first extent, the macroscopic ductility of the dynamically tested specimens is not affected by the presence of a notch. In fact, while the quasi-static ductility (necking strain) of a notched specimen drops tremendously with respect to that of a smooth specimen (Fig. 1) our results show that this is not the case for the dynamic tests (Appendix). Here, the sample elongation of both smooth and notched specimens reaches values of 4-5\%, which are definitely comparable (perhaps slightly higher) to the quasi-static necking strain values of smooth specimens. This result shows that, in addition to skipping it, the notch does not affect the structural ductility of the specimen.

Finally, while the results shown here are experimental, it is clear that future work should concentrate on capturing the observed effects in a numerical model that will allow optimized design of such impacted slender structures. This is work under progress.

\section{Conclusions}

Dynamic tensile testing of ductile steel specimens, with and without sharp notches, has been systematically carried out, keeping in mind the development of guidelines for the design of impacted structures. The main conclusions of this work can be summarized as follows:

- The location of the neck is deterministic and not random.

- The presence of sharp geometrical imperfections does not necessarily dictate the locus of the neck inception. The latter may skip the notch under suitable impact velocities.
Fig. 9 Tested sided notched specimens

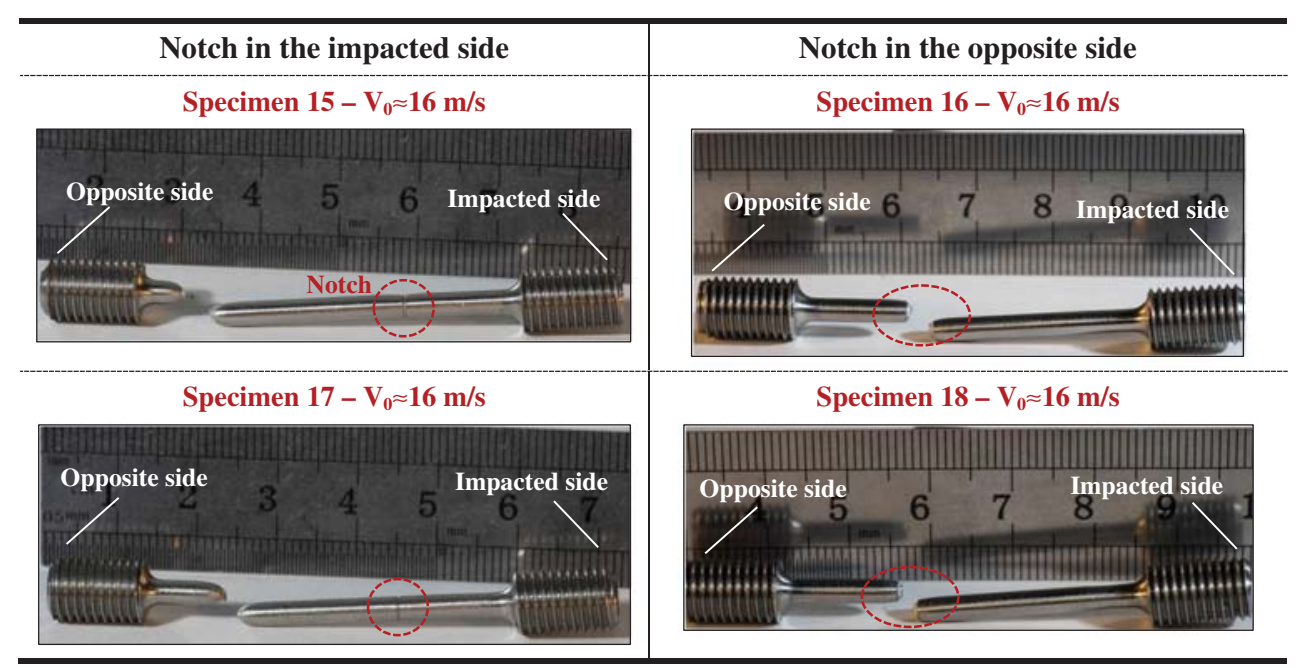


- The interaction of stress waves causes high gradients of strains along the sample. As a consequence, plastic strains may pile up to a critical level in the smooth section which overrides any stress concentration effects.

- In the present investigation, one can observe that the dynamic ductility of the specimen (necking strain) is not reduced by the presence of the notch.

- Consequently, unlike the quasi-static case, a straightforward extension of the weakest point assumption to dynamically deforming ductile materials is contradicted by the present experimental observations.

Acknowledgments D. Rittel acknowledges the support of Carlos III University with a Cátedra de Excelencia funded by Banco Santander during academic year 2011-2012.

The researchers of the University Carlos III of Madrid are indebted to the Ministerio de Ciencia e Innovación de España (Projects DPI/201124068 and DPI/2011-23191) for the financial support.

The authors thank Mr. A. Godinger for his technical support.

\section{Appendix - Video sequence of selected dynamic tensile tests}

Specimen 2-Smooth sample - Necking in the impacted side

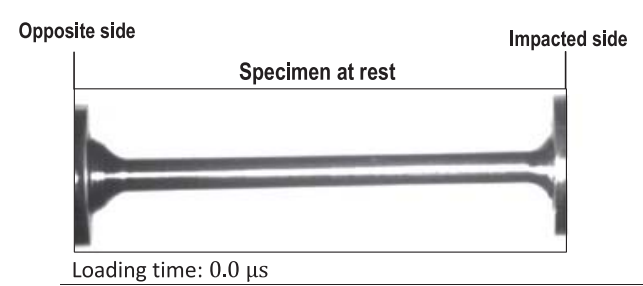

Homogeneous sample deformation

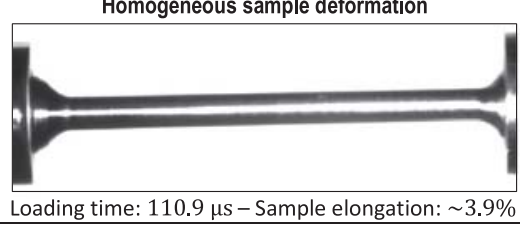

Necking development

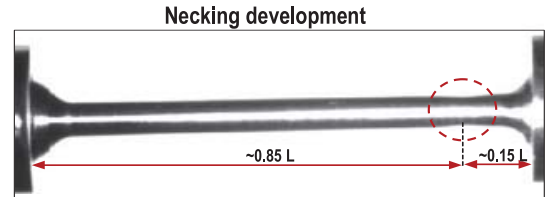

Loading time: $175.8 \mu \mathrm{s}-$ Sample elongation: $\sim 6.1 \%$

Fig. 10 Video sequence corresponding to specimen 2
Specimen 4-Smooth sample - Necking in the opposite side

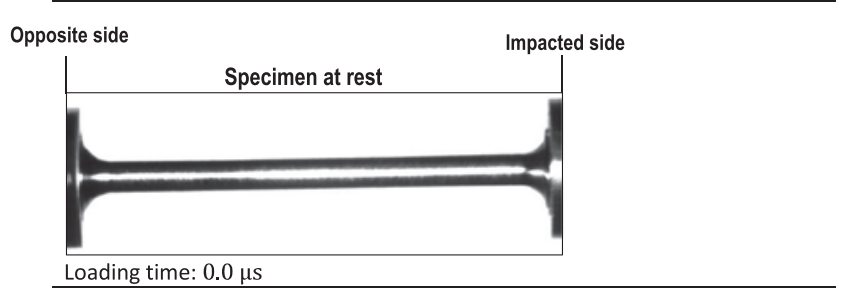

Homogeneous sample deformation

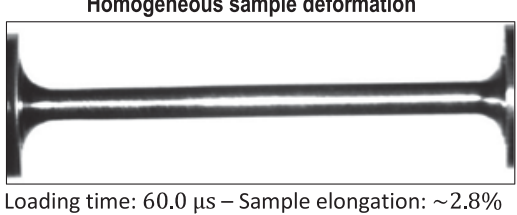

Onset of necking

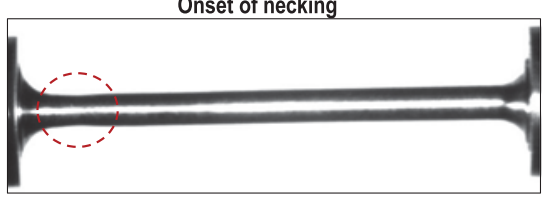

Loading time: $94.4 \mu$ s - Sample elongation: $\sim 4.0 \%$

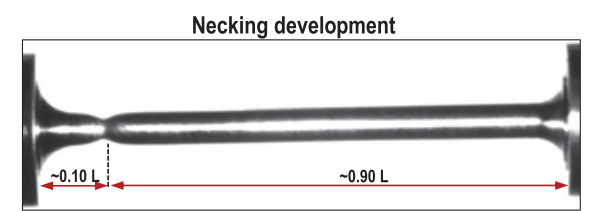

Loading time: $151.0 \mu \mathrm{s}-$ Sample elongation: $\sim 6.5 \%$

Fig. 11 Video sequence corresponding to specimen 4

Specimen 6 - Centrally notched sample - Necking in the impacted side

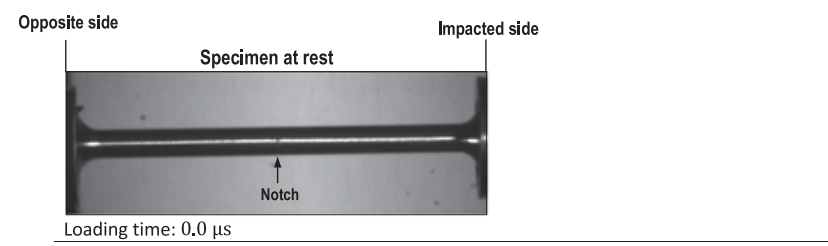

Homogeneous sample deformation

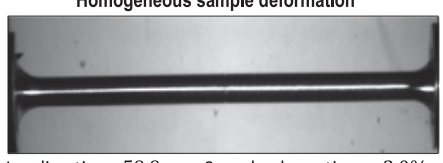

Loading time: $59.9 \mu \mathrm{s}-$ Sample elongation: $\sim 2.0 \%$

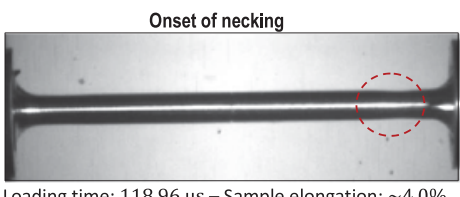

Loading time: $118.96 \mu \mathrm{s}-$ Sample elongation: $\sim 4.0 \%$

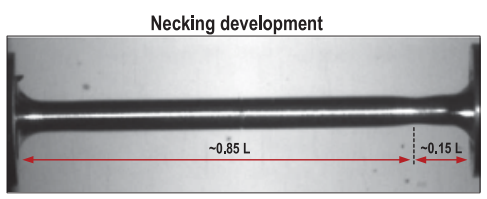

Loading time: $138.6 \mu \mathrm{s}$-Sample elongation: $\sim 4.7 \%$

Fig. 12 Video sequence corresponding to specimen 6 
Specimen 9-Centrally notched sample - Necking in the opposite side
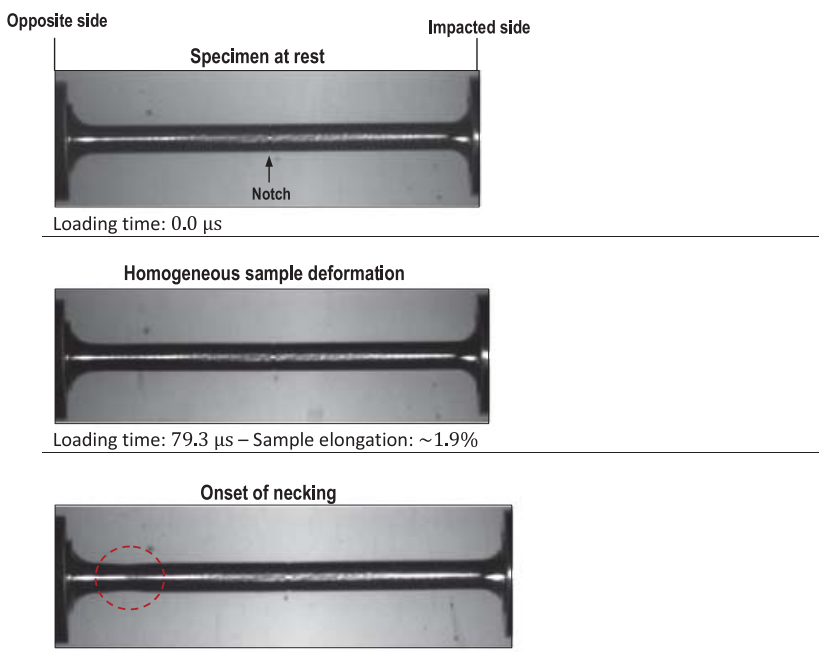

Loading time: $138.1 \mu \mathrm{s}-$ Sample elongation: $\sim 3.3 \%$

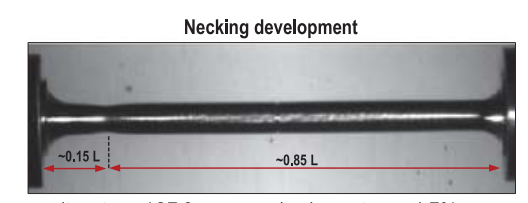

Loading time: $197.0 \mu \mathrm{s}$ - Sample elongation: $\sim 4.7 \%$

Fig. 13 Video sequence corresponding to specimen 9

Specimen 13 - Centrally notched sample - Necking in the notch
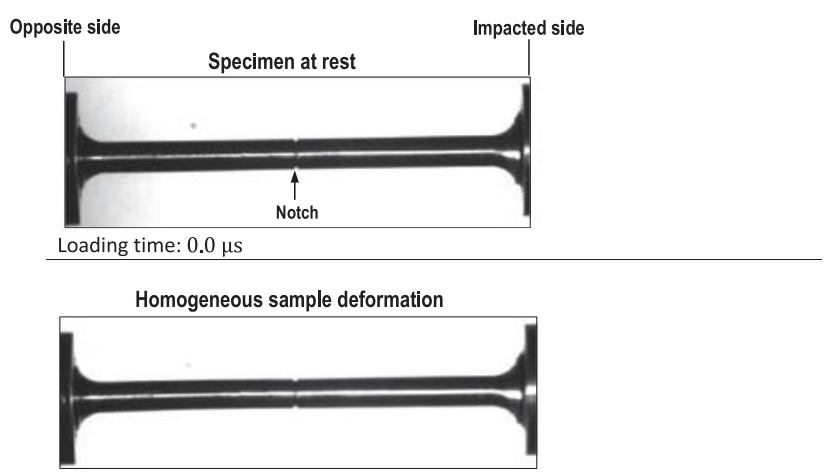

Loading time: $80.2 \mu \mathrm{s}$-Sample elongation: $\sim 3.2 \%$

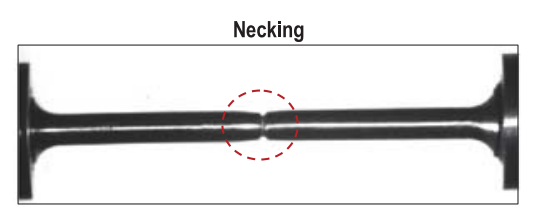

Loading time: $119.8 \mu \mathrm{s}$ - Sample elongation: $\sim 4.9 \%$

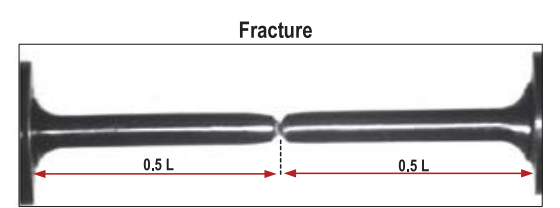

Loading time: $139.6 \mu \mathrm{s}$ - Sample elongation: $\sim 5.7 \%$

Fig. 14 Video sequence corresponding to specimen 13
Specimen 16 - Sided notched sample - Necking in the opposite side/notch

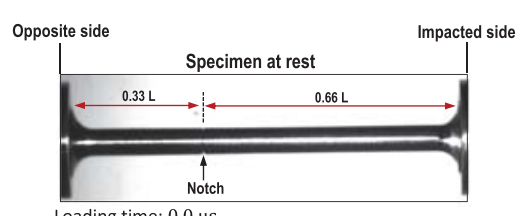

Loading time: $0.0 \mu \mathrm{s}$
Homogeneous sample deformation
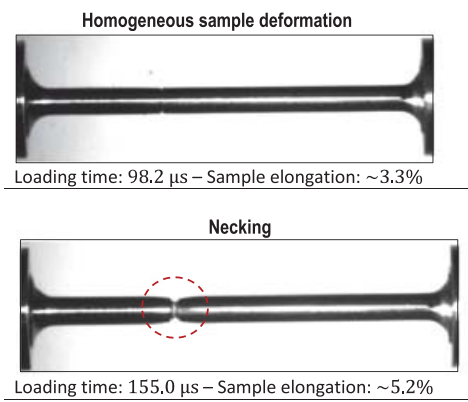

Loading time: $155.0 \mu \mathrm{s}-$ Sample
Fracture

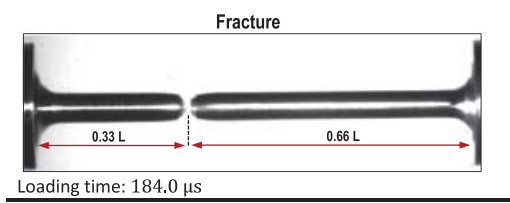

Fig. 15 Video sequence corresponding to specimen 16

Specimen 17 - Sided notched sample - Necking in the opposite side
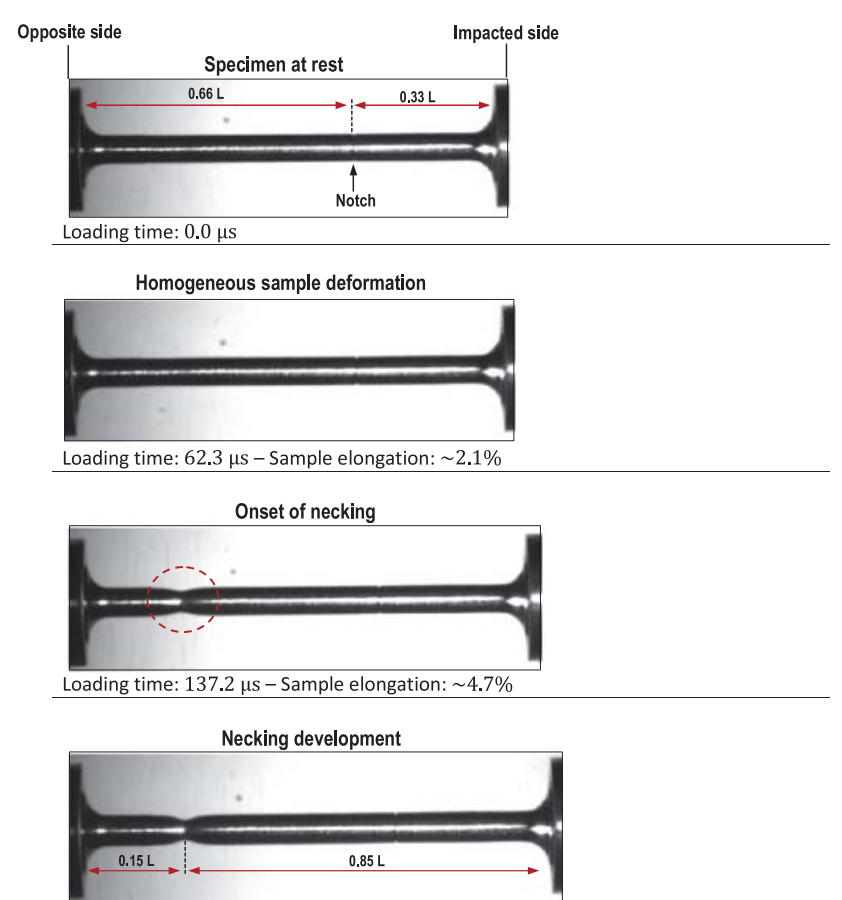

Loading time: $162.3 \mu \mathrm{s}$-Sample elongation: $\sim 5.5 \%$

Fig. 16 Video sequence corresponding to specimen 17 


\section{References}

1. Inglis CE (1913) Stresses in a plate due to the presence of cracks and sharp corners, Spring meetings of the fifty-fourth session of the Institution of Naval Architects

2. Neuber H (1936) Zur theorie der technischen formzahl. Forsch Ingenieurwes 7:6,271-6,274

3. Neuber H (1958) Theory of notch stresses. Springler, Berlin

4. Peterson RE (1933) Stress-Concentration Phenomena in Fatigue of Metals. ASME Trans APM-55-19

5. Peterson RE (1953) Stress concentration design factors. John Wiley and Sons, New York

6. Preuss E (1913) Forschungsarbeiten (Mitteilungen über Forschungsarbeiten), V.D.I., no. 126, 1912, and no. 134

7. Coker EG (1912-1913) The effects of holes and semi-circular notches on the distribution of stress in tension members. Proc Phys Soc 25: 1912-1913

8. Coker EG, Chakko KC, Satake Y (1919-1920) Photoelastic and strain measurements of the effects of circular holes on the distribution of stress in tension members. Proc Instn Engrs Shipbuild Scotl p. 34

9. Howland RCJ (1929) On the stresses in the neighborhood of a circular hole in a strip under tension. Phil Trans R Soc London A 229:49

10. Strandberg M (2001) Upper bounds for the notch intensity factor for some geometries and their use in general interpolation formulae. Eng Fract Mech 68:577-585

11. Zappalorto M, Lazzarin P (2011) Strain energy-based evaluations of plastic notch stress intensity factors at pointed V-notches under tension. Eng Fract Mech 78:2691-2706

12. Altenhof W, Zamani N, North W, Arnold B (2004) Dynamic stress concentrations for an axially loaded strut at discontinuities due to an elliptical hole or double circular notches. Int J Impact Eng 30(3)2: 255-274
13. James WG, North WPT (1969) Dynamic stress concentration using the photoelastic technique. J Strain Anal 4:261-266

14. Nakayama N, Ohashi M, Takeishi H (1998) Dynamic stress concentration in a strip plate with fillet. Int J Jpn Soc Mech Eng Ser A 41: 326-331

15. Matsumoto H, Adachi T, Kakuhama Y, Fukuzawa K (1990) Analysis of the dynamic stress concentration factor by the two-dimensional boundary element method. Int J Jpn Soc Mech Eng Ser A 33:37-43

16. Gerlach R, Sathianathan SK, Siviour C, Petrinic N (2011) A novel method for pulse shaping of Split Hopkinson tensile bar signals. Int $\mathrm{J}$ Impact Eng 38:976-980

17. Zhang H, Ravi-Chandar K (2006) On the dynamics of necking and fragmentation - I. Real-time and post-mortem observations in $\mathrm{Al}$ 6061-O. Int J Fract 142:183-217

18. Zhang H, Ravi-Chandar K (2008) On the dynamics of necking and fragmentation - II. Effect of material properties, geometrical constraints and absolute size. Int J Fract 150:3-36

19. Osovski S, Rittel D, Rodríguez-Martínez JA, Zaera R (2013) Dynamic tensile necking: influence of specimen geometry and boundary conditions. Mech Mater 62:1-13

20. Rusinek A, Zaera R, Klepaczko JR, Cheriguene R (2005) Analysis of inertia and scale effects on dynamic neck formation during tension of sheet steel. Acta Mater 53:5387-5400

21. Sørensen NJ, Freund LB (2000) Unstable neck formation in a ductile ring subjected to impulsive radial loading. Int J Solids Struct 37: 2265-2283

22. Rodríguez-Martínez JA, Vadillo G, Zaera R, Fernández-Sáez J (2013) On the complete extinction of selected imperfection wavelengths in dynamically expanded ductile rings. Mech Mater 60:107120

23. Rodríguez-Martínez JA, Rusinek A, Arias A (2009) Relation between strain hardening of steel and critical impact velocity in tension. J Theor Appl Mech 47:645-665 